\title{
Application of a Microstructure-Based ISV Plasticity Damage Model to Study Penetration Mechanics of Metals and Validation through Penetration Study of Aluminum
}

\author{
Yangqing Dou, ${ }^{1}$ Yucheng Liu, ${ }^{1}$ Youssef Hammi, ${ }^{2}$ and Wilburn Whittington ${ }^{2}$ \\ ${ }^{1}$ Department of Mechanical Engineering, Mississippi State University, Mississippi State, MS 39762, USA \\ ${ }^{2}$ Center of Advanced Vehicular Systems, Mississippi State University, Mississippi State, MS 39762, USA \\ Correspondence should be addressed to Yucheng Liu; liu@me.msstate.edu
}

Received 19 August 2016; Revised 21 October 2016; Accepted 31 October 2016; Published 29 January 2017

Academic Editor: Chung-Souk Han

Copyright ( $) 2017$ Yangqing Dou et al. This is an open access article distributed under the Creative Commons Attribution License, which permits unrestricted use, distribution, and reproduction in any medium, provided the original work is properly cited.

A developed microstructure-based internal state variable (ISV) plasticity damage model is for the first time used for simulating penetration mechanics of aluminum to find out its penetration properties. The ISV damage model tries to explain the interplay between physics at different length scales that governs the failure and damage mechanisms of materials by linking the macroscopic failure and damage behavior of the materials with their micromechanical performance, such as void nucleation, growth, and coalescence. Within the continuum modeling framework, microstructural features of materials are represented using a set of ISVs, and rate equations are employed to depict damage history and evolution of the materials. For experimental calibration of this damage model, compression, tension, and torsion straining conditions are considered to distinguish damage evolutions under different stress states. To demonstrate the reliability of the presented ISV model, that model is applied for studying penetration mechanics of aluminum and the numerical results are validated by comparing with simulation results yielded from the JohnsonCook model as well as analytical results calculated from an existing theoretical model.

\section{Introduction}

High-speed impact and penetration problems include large deformation, erosion, high strain-rate dependent nonlinear material behavior, and fragmentation. Therefore they have been a considerable research topic critical to the development of civil and military ballistic protection systems. Besides experimental approaches, use of scalable computers provides a promising, safe, and cost effective approach to investigate the high-rate damage process and evaluate material behavior during those processes while minimizing the need of actual expensive and destructive damage tests. Thus, it is imperative to develop very effective constitutive and computational models and approaches that can correctly predict strength, damage initiation, and failure progression of materials during high-rate damage.

Since the 1980s, a variety of constitutive models have been developed to model failure mechanics of materials and the damage processes. Among these models, the Johnson-Cook
(JC) constitutive model first presented in $1983[1,2]$ and its modified versions are being integrated into commercial finite element analysis (FEA) solvers such as LS-DYNA and ABAQUS for simulation of the high-rate damage process including high-speed impact and penetration. As a purely empirical, temperature and strain-rate dependent model, the JC model is the most widely used model for computational study of penetration mechanics of different metallic materials. For example, it was employed by Dey et al. [3] for assessing the ballistic perforation resistance of double-layered steel plates impacted by blunt and ogival projectiles. Kiliç and Ekici [4] determined ballistic limit of $500 \mathrm{HB}$ armor steel against $7.62 \mathrm{~mm}$ hardened steel core ammunition using the JC model implemented into LS-DYNA and validated their results by comparing to experimental data. In their study, the threshold penetration velocity for the bullet to penetrate a steel plate with $12.4 \mathrm{~mm}$ yielded from the JC model was found to be about $775 \mathrm{~m} / \mathrm{s}$, while the experimental data is $782 \mathrm{~m} / \mathrm{s}$, which are very close to each other. Manes et al. [5] studied the 
ballistic resistance of 6061-T6 aluminum plates subjected to a normal impact of small caliber armor piercing bullets using this model through both LS-DYNA and ABAQUS solver. Following the same approach, the researchers also studied the impact response of aluminum tubes subjected to impact of soft-core ball projectiles [6]. Zhang et al. used the JC theory to model titanium alloy TC4 and evaluated its ballistic penetration resistance through LS-DYNA [7]. Dou et al. also employed the JC model to capture the plasticity damage of a copper target plate penetrated by a nickel projectile and predicted its ballistic velocity [8]. Besides the penetration of single-layered plates, the JC model was also used by FloresJohnson et al. for numerical investigation of the ballistic performance of double- and triple-layered metallic plates made of either steel or aluminum impacted by a $7.62 \mathrm{~mm}$ APM2 projectile in a velocity range of $775-950 \mathrm{~m} / \mathrm{s}$ [9]. All the previous studies verified that, for commonly used impact protection and energy absorption metallic materials such as steels, aluminum alloys, and titanium alloys, the JC model is an accurate and effective numerical tool to simulate their high-speed impact and penetration process.

During the past 20-30 years, substantial effort has been invested in order to physically understand and mathematically describe the plasticity mechanisms that govern the penetration behavior of materials and to develop physically based material models based on those understandings. Among the developed physically based models, a microstructurebased internal state variable (ISV) plasticity damage model, Bammann-Chiesa-Johnson (BCJ) plasticity model [10], has been growing to a promising method for damage mechanics modeling and simulation. The BCJ model was later modified to include pore nucleation, pore growth, and pore coalescence evolution equations to account for stress state dependent damage evolution and to include the heterogeneities of microstructure for damage progression and failure analysis. The modified model has been successfully calibrated and applied for capturing the plasticity-damage evolution for a large variety of metallic alloys under different strain rates and loading conditions. One virtue of this study is to extend application of the developed ISV damage model for simulation of high-speed penetration process of metals, in which the strain rate can reach up to $\sim 10^{9} / \mathrm{s}$.

In order to validate this ISV damage model, it is employed to study the penetration mechanics of aluminum (Al) and the simulation results will be compared with those generated from the JC model, which has been proved to be correct by many previous studies. As a most-used metal, Al has been thoroughly tested in high-speed penetration tests and its penetration behavior has been observed from many previous tests [11]. Based on the large amount of experimental data, an analytical model of high accuracy was presented by Rosenberg and Forrestal [12] and verified by Piekutowski et al. [13] to be able to correctly predict the penetration performance of Al. Therefore, the numerical results calculated from the ISV damage model will again be compared with the analytical results to further validate its applicability for high-speed penetration study.

\section{Johnson-Cook Model}

The JC model describes constitutive relations of materials from their stress-strain behavior with strain-rate dependence and even temperature sensitivity. Verified by previous experimental-computational studies, the JC constitutive model is best suited for modeling materials that are subjected to high strain rate deformation, as shown in

$$
\begin{aligned}
\sigma= & {\left[A+B\left(\varepsilon_{p}\right)^{n}\right]\left[1+C \ln \left(\frac{\dot{\varepsilon}_{p}}{\dot{\varepsilon}_{0}}\right)\right] } \\
& \cdot\left[1-\left(\frac{T-T_{a}}{T_{f}-T_{a}}\right)^{m}\right] .
\end{aligned}
$$

In (1), $A$ is the elastic limit; $B$ and $n$ are the characteristic constants of the hardening behavior; $C$ denotes the sensitivity to the strain rate; $\dot{\varepsilon}_{0}$ is the reference strain rate (typically set to $1 / s) ; \varepsilon_{p}$ and $\dot{\varepsilon}_{p}$ represent the plastic strain and plastic strain rate, respectively; $T, T_{a}, T_{f}$ are, respectively, the actual temperature, room temperature $(293 \mathrm{~K})$, and the melting absolute temperature; $m$ is a material constant for the temperature tendency. As demonstrated by Dey et al. [3], the constants $A, B$, and $n$ have to be measured and calibrated through a series of tensile tests. The constants $C$ and $n$ have to be decided through a set of split Hopkinson pressure bar tests.

Within the JC model framework, the impact and penetration on ductile metal is reliably predicted by the accumulated equivalent plastic strain; therefore, the JC damage model predicts the failure and damage of materials based on their plastic strain. In that model, the equivalent strain to fracture $\varepsilon_{f}$ is defined by

$$
\begin{aligned}
& \varepsilon_{f} \\
& =\left[D_{1}+D_{2} \exp \left(D_{3} \frac{\sigma_{H}}{\sigma_{\mathrm{vm}}}\right)\right]\left[1+\frac{\dot{\varepsilon}_{p}}{\dot{\varepsilon}_{0}}\right]^{D_{4}}\left[1+D_{5} T^{*}\right],
\end{aligned}
$$

where $D_{1}$ to $D_{5}$ are the material constants, $\sigma_{H}$ is the hydrostatic stress, $\sigma_{\mathrm{vm}}$ is the Von Mises equivalent stress, $\sigma_{h} / \sigma_{\mathrm{vm}}$ represents stress taxability, and $T^{*}$ is the homologous temperature.

Bao-Wierzbicki (B-W) criterion [14] is selected as the failure criterion because such criterion can describe the failure onset at different stress triaxiality with good accuracy and reduced computational efforts. It is assumed that the selected $\mathrm{B}-\mathrm{W}$ criterion is to be uncoupled from the constitutive model (see (1)). The failure condition will be checked at each step for each finite element outside the algorithm of the stress and strain calculation. Equation (3) displays the criterion, where a damage parameter $\omega_{D}$ (referred to each finite element) is based on a cumulative law and grows as a function of the accumulated plastic strain as

$$
\omega_{D}\left(\bar{\varepsilon}_{p}\right)=\int_{0}^{\bar{\varepsilon}_{p}} \frac{d \varepsilon_{p}}{\varepsilon_{f}\left(\sigma_{h} / \sigma_{\mathrm{vm}}, \dot{\varepsilon}_{p}, T\right)},
$$

where $\varepsilon_{f}$ is the strain at failure as defined in (2), which is a function of the absolute temperature $T$, the plastic strain rate, 
and the stress triaxiality $\sigma_{h} / \sigma_{\mathrm{vm}}$. By adopting such criterion, the damage is not coupled with the JC constitutive model and there is no effect on the analysis before $\omega_{D}$ reaches the critical value (conventionally calibrated at 1 ). During the simulation, an element is damaged when $\omega_{D}=1$ and failed elements suddenly fail and completely lose their load-carrying capability. The failed elements and related nodes are therefore removed from the analysis.

The JC constitutive and damage models are combined with the B-W failure criterion to jointly provide a framework for penetration modeling and simulation. The accuracy of such framework had been validated by Kiliç and Ekici in literature [4]. In their study, the same formulation (see (1)-(3)) was used to investigate ballistic resistance of high hardness armor steels against $7.62 \mathrm{~mm}$ armor piercing ammunition. The numerical results agreed very well with the experimental data. For example, according to their simulation results, the ballistic limit velocity for the $12.4 \mathrm{~mm}$ thick armor steel plate was found to be about $775 \mathrm{~m} / \mathrm{s}$, while that velocity was measured as $782 \mathrm{~m} / \mathrm{s}$ from ballistic tests conducted by Demir et al. [15], which are very close. Therefore, it should be convincing that the simulation results yielded from the JC model can be used as a benchmark to validate the accuracy of the present microstructure-based ISV model in modeling and computing damage behavior of materials during the high-speed penetration process.

\section{The Microstructure-Based ISV Plasticity Damage Model}

In the revised BCJ model, a set of ISV rate equations are cast in a continuum framework to model void nucleation, growth, and coalescence during the damage process of materials. The framework of this microstructure-based ISV model is described as follows:

$$
\stackrel{o}{\sigma}=\lambda(1-\phi) \operatorname{tr}\left(\underline{D}^{e}\right) \underline{I}+2 \mu(1-\phi) \underline{D}^{p}-\left(\frac{\dot{\phi}}{1-\phi}\right) \underline{\sigma} .
$$

In the above equation the Cauchy stress and its corotational rate are denoted by $\underline{\sigma}$ and $\stackrel{o}{\sigma}$, respectively; $\phi$ is an ISV that indicates the damage state with $\dot{\phi}$ representing its variation with respect to time; $\lambda$ and $\mu$ are the elastic Lame constants; $D^{e}$ is the elastic deformation tensor; and $I$ is the second-order identity tensor. The underscore symbol indicates a second rank tensor. $\phi$ equals 0 indicates that there is no damage in the material, while when $\phi$ reaches 1 means that the material or structural components have fully failed. In (4), $\underline{D}^{e}$ and $\dot{\phi}$ are the unknown terms.

For a simple material model, it is assumed that $\underline{D}^{e}=\underline{D}-$ $\underline{D}^{p}$, where $\underline{D}^{p}$ is an ISV representing the plastic deformation tensor, which is given as

$$
\begin{gathered}
\underline{D}^{p}=f(T) \times \sinh \left\{\frac{\left\|\underline{\sigma}^{\prime}-\underline{\alpha}\right\|-[R+Y(T)](1-\phi)}{V(T)(1-\phi)}\right\} \\
\cdot \frac{\underline{\sigma}^{\prime}-\underline{\alpha}}{\left\|\underline{\sigma}^{\prime}-\underline{\alpha}\right\|},
\end{gathered}
$$

where $\sigma^{\prime}$ is the deviatoric part of stress tensor, $T$ is the absolute temperature, $\underline{\alpha}$ is the kinematic hardening (an ISV identifying the effect of anisotropic dislocation density), and $R$ is the isotropic hardening (an ISV identifying the effect of global dislocation density). Functions $V(T), Y(T)$, and $f(T)$ are related to yielding with Arrhenius-type temperature dependence. Specifically, $V(T)$ determines the magnitude of rate dependence on yielding, $f(T)$ determines when the rate dependence affects initial yielding, and $Y(T)$ is the rateindependent yield stress. Equation (6) gives forms for those functions as

$$
\begin{aligned}
& V(T)=C_{1} e^{\left(-C_{2} / T\right)}, \\
& Y(T)=C_{3} e^{\left(-C_{4} / T\right)}, \\
& V(T)=C_{5} e^{\left(-C_{6} / T\right)},
\end{aligned}
$$

where $C_{1}$ to $C_{6}$ are material parameters related to the yield stress, which can be calibrated from isothermal compression tests at different temperature and strain rate levels.

Two more time derivative equations related to $R$ and $\underline{\alpha}$ are needed to solve for $\underline{D}^{p}$. The corotational rate of the kinematic hardening $\stackrel{o}{\alpha}$ and the material time derivative of isotropic hardening $\dot{R}$ are represented in a hardening-recovery format as

$$
\begin{aligned}
& \underline{o}=\left\{h(T) \underline{D}^{p}-\left[\sqrt{\frac{2}{3}} r_{d}(T)\left\|\underline{D}^{p}\right\|+r_{s}(T)\right]\|\underline{\alpha}\| \underline{\alpha}\right\}, \\
& \dot{R}=\left\{H(T) \underline{D}^{p}-\left[\sqrt{\frac{2}{3}} R_{d}(T)\left\|\underline{D}^{p}\right\|+R_{s}(T)\right] R^{2}\right\} .
\end{aligned}
$$

Two types of recovery, dynamic recovery and static recovery, coexist in the dislocation populations and morphology within crystallographic materials. In $(7), r_{d}(T)$ and $R_{d}(T)$ are scalar functions of temperature describing dynamic recovery whereas $r_{s}(T)$ and $R_{s}(T)$ are scalar functions describing thermal (static) recovery. $h(T)$ and $H(T)$ denote anisotropic and isotropic hardening modulus in those equations, respectively. The recovery and hardening modulus functions are defined by Solanki et al. [16] as

$$
\begin{aligned}
& r_{d}(T) \\
& \quad=C_{7}\left[1+C_{a}\left(\frac{4}{27}-\frac{J_{3}^{\prime 2}}{J_{2}^{\prime 3}}\right)-C_{b}\left(\frac{J_{3}^{\prime}}{J_{2}^{\prime 3 / 2}}\right)\right] e^{\left(-C_{8} / T\right)}, \\
& R_{d}(T) \\
& \quad=C_{13}\left[1+C_{a}\left(\frac{4}{27}-\frac{J_{3}^{\prime 2}}{J_{2}^{\prime 3}}\right)-C_{b}\left(\frac{J_{3}^{\prime}}{J_{2}^{\prime 3 / 2}}\right)\right] e^{\left(-C_{14} / T\right)}, \\
& r_{s}(T)=C_{11} e^{\left(-C_{12} / T\right)}, \\
& R_{s}(T)=C_{17} e^{\left(-C_{18} / T\right)},
\end{aligned}
$$


$h(T)$

$$
\begin{aligned}
= & C_{9}\left[1+C_{a}\left(\frac{4}{27}-\frac{J_{3}^{\prime 2}}{J_{2}^{\prime 3}}\right)+C_{b}\left(\frac{J_{3}^{\prime}}{J_{2}^{\prime 3 / 2}}\right)\right] e^{\left(-C_{8} / T\right)} \\
& -C_{10} T
\end{aligned}
$$

$H(T)$

$$
\begin{aligned}
= & C_{15}\left[1+C_{22}\left(\frac{4}{27}-\frac{J_{3}^{\prime 2}}{J_{2}^{\prime 3}}\right)+C_{b}\left(\frac{J_{3}^{\prime}}{J_{2}^{\prime 3 / 2}}\right)\right] e^{\left(-C_{8} / T\right)} \\
& -C_{16} T
\end{aligned}
$$

where $J_{2}^{\prime}=(1 / 2)\left(\underline{\sigma}^{\prime}-\underline{\alpha}^{\prime}\right)^{2}, J_{3}^{\prime}=(1 / 3)\left(\underline{\sigma}^{\prime}-\underline{\alpha}^{\prime}\right)^{3}, C_{7}$ to $C_{12}$ are the material plasticity parameters related to kinematic hardening and recovery terms, $C_{13}$ to $C_{18}$ are the material plasticity parameters related to isotropic hardening and recovery terms, and $C_{a}$ and $C_{b}$ are the material plasticity parameters related to dynamic recovery and anisotropic hardening terms, respectively. Constants $C_{1}$ to $C_{18}$ are determined from macroscale experiments at different temperatures and strain rates.

In FEA, the variable $\phi$ represents the damage fraction of material within a continuum element. The mechanical properties of a material depend upon the amount and the type of microdefects within its structure. Deformation changes these microdefects, and when the number of microdefects accumulates, damage is said to have grown. Based on fracture mechanics and microscale physical observations, the damage is governed by three components of damage progression mechanisms, which are void nucleation $(\eta)$, growth $(\nu)$, and coalescence $(c)$ from particles and pores in the material. The rates of void nucleation and void growth are expressed by Solanki et al. [16] as

$$
\begin{aligned}
& \dot{\eta}=\left\|\underline{D}^{p}\right\| \frac{C_{\text {coeff }} d^{1 / 2}}{K_{I C} f^{1 / 3}} \\
& \cdot \eta\left[a\left(\frac{4}{27}-\frac{J_{3}^{2}}{J_{2}^{3}}\right)+b \frac{J_{3}}{J_{2}^{3 / 2}}+c\left\|\frac{I_{1}}{\sqrt{J_{2}}}\right\|\right] e^{\left(C_{\eta T} / T\right)}, \\
& \dot{v}=\frac{\sqrt{3} R_{0}}{2(1-m)}\left[\sinh \left(\sqrt{3}(1-m) \frac{\sqrt{2} I_{1}}{3 \sqrt{J_{2}}}\right)\right]\left\|\underline{D}^{p}\right\| .
\end{aligned}
$$

$C_{\text {coeff }}$ in (9) is a material constant that considers the void nucleation response as a function of initial conditions; $d$ is the particle size; $K_{I C}$ represents the fracture toughness; $f$ denotes the volume fraction of second phase particles; $C_{\eta T}$ is a temperature dependent parameter related to void nucleation; $I_{1}$, $J_{2}$, and $J_{3}$ are the independent overstress invariants; $m$ is void growth constant; $R_{0}$ is the initial void radius; $a, b$, and $c$ are the material constants related to void nucleation, which should be calibrated from torsion, tension, and compression tests, respectively. A void coalescence model is also introduced as a function of $\eta$ and $\nu$ as

$$
\dot{c}=\left[C d_{1}+C d_{2}(\dot{\eta} \nu+\dot{\nu} \eta)\right] e^{\left(C_{\mathrm{CT}}\right)} .
$$

TABle 1: Parameters of Al for calibration.

\begin{tabular}{lc}
\hline Parameters & Value \\
\hline Shear modulus $(G)$ & $25.56 \mathrm{GPa}$ \\
Bulk modulus (Bulk) & $66.67 \mathrm{GPa}$ \\
Melt temperature $(\mathrm{K})$ & $933.52^{\circ} \mathrm{F}$ \\
Heat generation term & 0.37 \\
Particle fracture toughness & 1000 \\
Particle McClintock damage constant & 0.3 \\
\hline
\end{tabular}

The parameters $C d_{1}$ and $C d_{2}$ are related to the first and second normalized nearest neighbor distance parameters, respectively, and $C_{\mathrm{CT}}$ is the void coalescence temperature dependent parameter.

The theoretical background of the present model is that the mechanical properties of a material depend upon the amount and type of microdefects within its structure. Deformation tends to change these microdefects, and when the number of the microdefects accumulates, the damage state is said to have grown. $\alpha, R, \sigma, \phi, \eta, \nu$, and $c$ in (4)-(11) complete the required ISVs in this microstructure-property relationship material constitutive model. Those ISVs correlate the microstructural characteristics of the material, such as particle size, particle volume fraction, pore/void radius, pore/void volume fraction, fracture toughness, and grain size, with the material stress-strain response and macroscale damage performance.

\section{Calibration of ISV Model Constants for Al}

In order to apply the ISV plasticity equations to model aluminum, the model constants need to be calibrated for that material. In order to facilitate the experimental calibration, software for curve fitting, DMGfit, was released at the Center of Advanced Vehicular Systems (CAVS) at Mississippi State University (MSU) [17]. That software has been synchronized with the latest version of the ABAQUS UMAT implementation of the ISV damage model so that the calibrated model constants can be directly merged by DMGfit into the "USER MATERIAL, CONSTANTS" section through the ABAQUS input deck. To calibrate and determine model constants for $\mathrm{Al}$, compression experiments were executed with initial temperature $289^{\circ} \mathrm{F}$ and strain rate of $1000 \mathrm{~s}^{-1}$, under different pressures. Shear modules, bulk modules, melt temperature, heat generation term, and other parameters of $\mathrm{Al}$ are tabulated in Table 1 and used for DMGfit. In order to calculate the constants for $\mathrm{Al}$, different sets of stress versus strain data are needed to collect during experiments.

The high-rate compression tests were executed using a split Hopkinson pressure bar (SPHB) at a strain rate of $1000 \mathrm{~s}^{-1}$. The specimens used for tests are Al cylinders with $6.4 \mathrm{~mm}$ diameter and $6.98 \mathrm{~mm}$ in length. In order to investigate mechanical properties of $\mathrm{Al}$, pressures of 150, 200, and 240 bar were applied to the same specimens at different temperatures. The highest pressure of 240 bar was chosen according to experimental practice implemented by Ledbetter and Reed [18] and employed in our previous study [19]. Five sets of stress-strain data were collected from the experiments and 


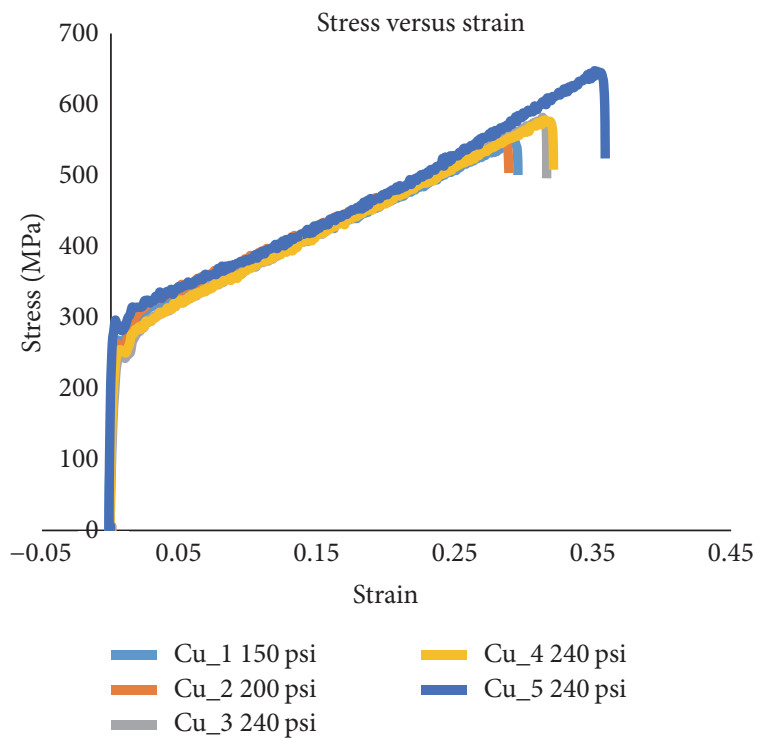

Figure 1: Stress-strain curves of aluminum under different pressures.

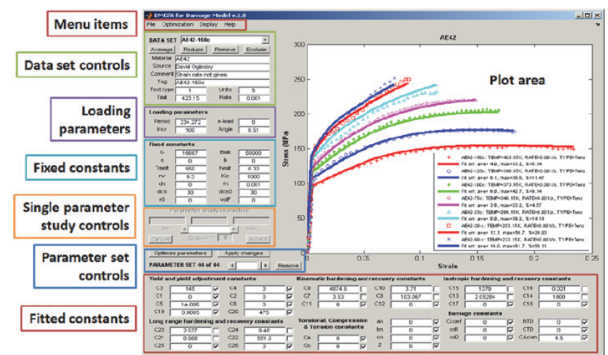

FIGURE 2: The logical groupings of controls in the DMGfit GUI.

saved as experimental data for DMGfit, as plotted in Figure 1. Those sets of data were obtained under 150, 200, and 240 bar and at initial temperatures $298.15^{\circ} \mathrm{F}$ and $542^{\circ} \mathrm{F}$, respectively.

The obtained experimental data will be loaded into DMGfit through its graphical user interface (GUI) to calibrate the missing material constants. Figure 2 displays a GUI example, which includes regions for experimental datasets; loading parameters; fixed constants; yield and yield adjustment constants; kinematic hardening and recovery constants; isotropic hardening and recovery constants; cyclic range hardening and recovery constants; torsional, compression, and tension constants; and damage constants. A typical procedure for curve fitting and calibrating constants using DMGfit is described as follows:

(1) Load all experimental datasets. For each dataset, establish the experiment settings (initial temperature, strain rate, stress units, etc.), loading parameters, and fixed constants.

(2) Start by fitting the dataset with the lowest temperature and lowest rate. If there are different tests, fit the compression datasets first, then tension datasets, and finally torsion datasets.
TABLE 2: Material properties and model constants for Al.

\begin{tabular}{|c|c|}
\hline \multicolumn{2}{|c|}{ Material properties } \\
\hline Young's modulus $(E)$ & $70 \mathrm{GPa}$ \\
\hline Density $(\rho)$ & $2700\left(\mathrm{~kg} / \mathrm{m}^{3}\right)$ \\
\hline Poisson's ratio $(\nu)$ & 0.33 \\
\hline \multicolumn{2}{|c|}{ Constants of JC model } \\
\hline$A$ & $265 \mathrm{MPa}$ \\
\hline$B$ & $426 \mathrm{MPa}$ \\
\hline C & 0.015 \\
\hline$n$ & 0.34 \\
\hline$m$ & 1.09 \\
\hline$D_{1}$ & 0.77 \\
\hline $\mathrm{D}_{2}$ & 1.45 \\
\hline$D_{3}$ & 0.47 \\
\hline$D_{4}$ & 0 \\
\hline$D_{5}$ & 0 \\
\hline \multicolumn{2}{|c|}{ Constants of ISV model } \\
\hline$C_{1}$ & 2.4 \\
\hline$C_{3}$ & 10 \\
\hline$C_{5}$ & 210 \\
\hline$C_{7}$ & 0.274742 \\
\hline$C_{9}$ & 511.843 \\
\hline$C_{11}$ & 0.000442617 \\
\hline$C_{13}$ & 1.16449 \\
\hline$C_{15}$ & 173.409 \\
\hline$C_{17}$ & 35.8129 \\
\hline
\end{tabular}

Other unlisted constants assume the value of " 0 ".

(3) For the first dataset, adjust the constants yield $\mathrm{C}_{3}$; kinematic hardening $\mathrm{C}_{9}$ and recovery $\mathrm{C}_{7}$; and isotropic hardening $C_{15}$ and recovery $C_{13}$.

(4) Then restore second dataset. If it has a different temperature than the first, adjust the constants $\left\{C_{3}, C_{4}\right\}$ if yield is temperature dependent, then $\left\{C_{10}, C_{8}, C_{16}\right.$, $\left.\mathrm{C}_{14}\right\}$. If the dataset has a different strain rate, adjust $C_{1}$ and $C_{5}$; if yield is strain rate dependent, then adjust $\left\{C_{9}, C_{7}, C_{11}\right\}$ and $\left\{C_{15}, C_{13}, C_{17}\right\}$.

(5) Repeat step (4) for the rest of the datasets. If adjusting the temperature dependence constants (even Cs) does not produce good models for high temperature data, adjust $C_{19}$ and $C_{20}$.

(6) Adjust damage constants. Readjust constants in other boxes as necessary.

(7) Record your results.

The calibrated material constants for $\mathrm{Al}$ can be found in Table 2.

\section{Numerical Simulation of Al-Al Penetration}

Next, to verify the applicability of the present microstructurebased ISV damage model on simulation of penetration process, an $\mathrm{Al}-\mathrm{Al}$ penetration process is modeled and computed with the present ISV model and the JC model separately, 
using ABAQUS/Explicit code. In this example, a $100 \mathrm{~mm}$ by $100 \mathrm{~mm}$ Al plate with a thickness of $25 \mathrm{~mm}$ will be penetrated by a $30 \mathrm{~mm}$ long $\mathrm{Al}$ rod with a diameter of $10 \mathrm{~mm}$ from its center. The thickness of $25 \mathrm{~mm}$ is selected for the $\mathrm{Al}$ plate so that the penetration through the thickness can be well observed through the simulations and the effects of the ductility of $\mathrm{Al}$ on its plasticity deformation during the penetration can be displayed.

In the FEA model, adaptive mesh strategy is employed to correctly capture the high plastic strain rate, high stress variation, large strain, and damage progression expected in impact and penetration zone while keeping the entire model computational cost effective. As shown in Figure 1, both plate and rod are modeled using eight node brick elements (C3D8R). As for the Al target plate model, element size in the impact/penetration zone is $0.5 \mathrm{~mm}$ while the element size for the remaining model is $10 \mathrm{~mm}$. Overall 2704 elements are defined for the plate by executing this mesh strategy. The Al rod model is meshed by 3744 elements with fine and coarse element size as $0.5 \mathrm{~mm}$ and $2 \mathrm{~mm}$, respectively. It is noteworthy that the grain size of the pure $\mathrm{Al}$ was measured as $16 \mu \mathrm{m}(0.016 \mathrm{~mm})$ in our lab. In other words, in order to correctly capture the penetration behavior of $\mathrm{Cu}$, only about 30 grains are defined along the length of a single element in the impact and penetration zone, which is considered a very fine mesh in a macroscale model. The FE Al-Al high-speed impact and penetration scenario is displayed in Figure 3, with total amount of 6448 brick elements at three different sizes, and all edges of the Al plate are fully constrained. The contact between the penetrator and the plate is modeled using the general contact algorithm of ABAQUS/Explicit. In addition, the option "interior" for the contact algorithm is activated in order to consider not only the exterior contact but also the contact of interior elements. Figures 3(a) and 3(b) display the FEA models the Al plate and Al rod, respectively.

The JC model and the present ISV damage model are used to define the properties for the Al material and numerical simulations are then carried off to compare the results yielded from the two material models. Material properties of $\mathrm{Al}$ and constants of the JC model and the ISV damage model are listed in Table 2. The JC model constants for Al had been calibrated by Champagne [20] and the ISV model constants were calibrated at the Center of Advanced Vehicular System (CAVS) at Mississippi State University.

\section{Numerical Results and Comparison}

Overall 26 simulations are conducted, in which the Al rod impacted and penetrated the $\mathrm{Al}$ target at a set of different initial velocities (from $100 \mathrm{~m} / \mathrm{s}$ to $1000 \mathrm{~m} / \mathrm{s}$ ) to (1) find the ballistic velocity that the $\mathrm{Al}$ rod is just about to penetrate through the target and (2) depict the penetration properties of $\mathrm{Al}$ in terms of the residual velocity and penetration time. As observed from the animation files shown in Figures 4 and 5, the JC model and the ISV damage model render a similar Al-Al penetration process (in order to save computing time, only a quarter model was simulated and displayed). Table 2 lists important results generated from the JC model and the present ISV damage model, including the initial and

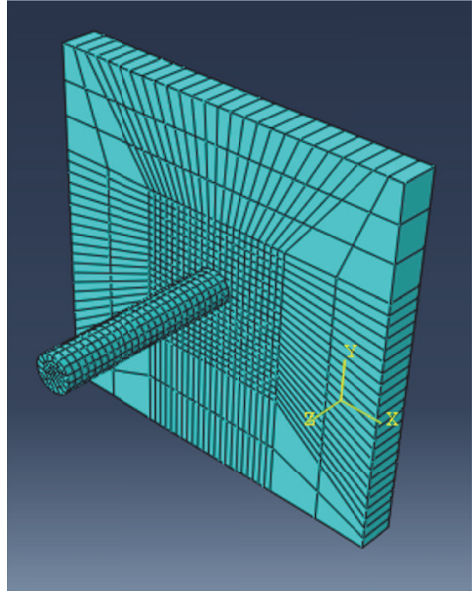

FIGURE 3: FEA model for the Al-Al penetration.

residual velocities of the $\mathrm{Al}$ rod, penetration time, a property that indicates the penetration resistance of a material, and $\left(v_{\text {initial }}^{2}-v_{\text {residual }}{ }^{2}\right) / v_{\text {initial }}{ }^{2}$, an indicator representing the amount and percentage of kinetic energy loss during the penetration process. Figures 6-8 compare those results. Some of the numerical results calculated from the JC model have been published in [21].

As shown in Table 3 and Figures 6-8, the penetration results obtained from the present ISV damage model are very close to what were generated from the JC model. The ballistic penetration velocity is found to be about $180 \mathrm{~m} / \mathrm{s}(179.93 \mathrm{~m} / \mathrm{s}$ from the ISV damage model and $179.97 \mathrm{~m} / \mathrm{s}$ from the JC model, which is very close).

\section{Analytical Validation}

In order to further validate the obtained numerical results and evaluate the accuracy of the present ISV damage model on penetration simulation, the simulation results are compared with analytical results calculated from Rosenberg and Forrestal's model [12]. Rosenberg and Forrestal presented a plate perforation model for nondeforming rods and target plates that experience ductile hole growth. That model uses the cylindrical, cavity-expansion approximation and represents the target as an elastic, perfectly plastic material. The accuracy of that model in predicting the penetration behavior of materials was verified by Piekutowski through a series of penetration experiments conducted with aluminum plates and aluminum rods [13]. According to Rosenberg and Forrestal, the ballistic penetration velocity for the presented cylindrical aluminum rod to just penetrate through the $25 \mathrm{~mm}$ thick aluminum plate can be estimated as

$$
v_{\mathrm{bl}}=\sqrt{2 \frac{t}{L} \frac{(Y / \sqrt{3})}{\rho_{\mathrm{rod}}}},
$$

where $t$ is thickness of the aluminum plate $(25 \mathrm{~mm}), L$ is the length of aluminum rod $(30 \mathrm{~mm}), Y$ is the yield stress of aluminum $(90 \mathrm{MPa})$, and $\rho_{\text {rod }}$ is the density of the aluminum penetrator $\left(2700 \mathrm{~kg} / \mathrm{m}^{3}\right)$. Substituting all the values into (12) 


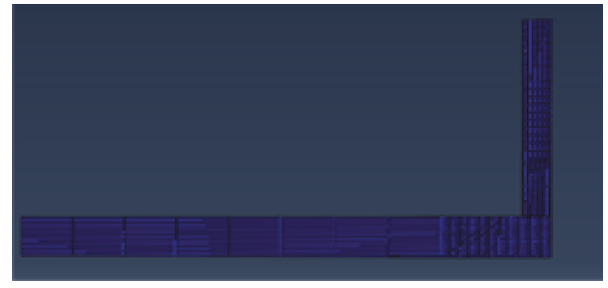

(a)

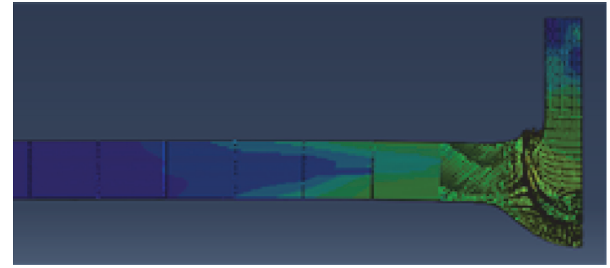

(c)

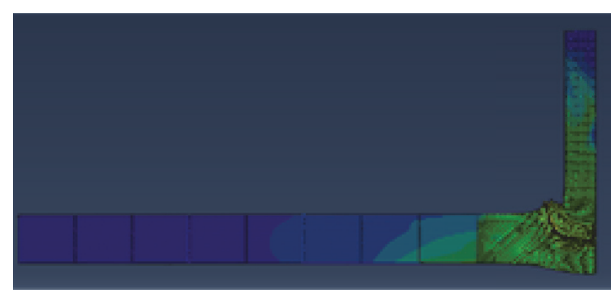

(b)

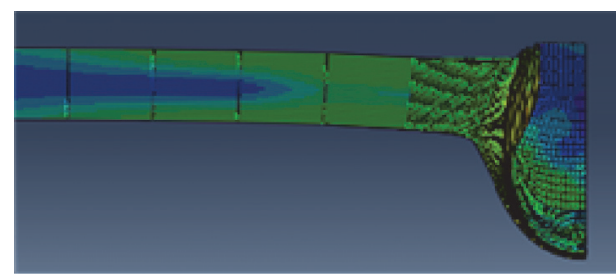

(d)

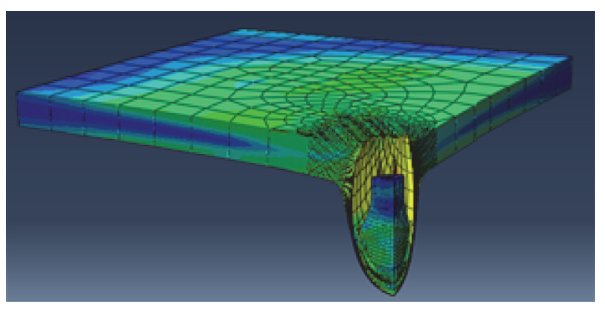

(e)

FIGURE 4: Penetration simulation yielded from JC model, $v_{\text {initial }}=200 \mathrm{~m} / \mathrm{s}$ at $t=0$ (a), $50 \mu \mathrm{s}(\mathrm{b}), 100 \mu \mathrm{s}(\mathrm{c}), 150 \mu \mathrm{s}(\mathrm{d})$, and $265 \mu \mathrm{s}(\mathrm{e})$.

TABLE 3: Initial velocities and residual velocities yielded from JC model and the ISV damage model.

\begin{tabular}{|c|c|c|c|c|c|c|}
\hline \multirow{2}{*}{$v_{\text {initial }}(\mathrm{m} / \mathrm{s})$} & \multicolumn{2}{|c|}{$v_{\text {residual }}(\mathrm{m} / \mathrm{s})$} & \multicolumn{2}{|c|}{ Penetration time $(\mu \mathrm{s})$} & \multicolumn{2}{|c|}{$\left(v_{\text {initial }}^{2}-v_{\text {residual }}{ }^{2}\right) / v_{\text {initial }}{ }^{2}(\%)$} \\
\hline & JC model & ISV model & JC model & ISV model & JC model & ISV model \\
\hline 170 & 0 & 0 & NA & NA & 100 & 100 \\
\hline 180 & 3 & 5 & 285 & 278 & 100 & 100 \\
\hline 190 & 12.5 & 16.5 & 277 & 269 & 99.57 & 99.25 \\
\hline 200 & 54.1 & 68 & 265 & 253 & 92.68 & 88.44 \\
\hline 300 & 220.7 & 251 & 120 & 95 & 45.88 & 30.00 \\
\hline 400 & 268.4 & 297 & 84 & 77 & 54.98 & 44.87 \\
\hline 500 & 337 & 405 & 66 & 59 & 54.27 & 34.39 \\
\hline 600 & 470.7 & 503.6 & 54 & 50 & 38.46 & 29.55 \\
\hline 700 & 571.7 & 601.2 & 48 & 41 & 33.30 & 26.24 \\
\hline 800 & 643.9 & 705.4 & 42 & 35 & 35.22 & 22.25 \\
\hline 1000 & 839.4 & 907.1 & 30 & 19 & 29.54 & 17.72 \\
\hline
\end{tabular}

the ballistic velocity $v_{\mathrm{bl}}$ can be calculated as $179.1 \mathrm{~m} / \mathrm{s}$, which is very close to the velocities obtained from both the ISV damage model and the JC model (about $180 \mathrm{~m} / \mathrm{s}$ ).

The relationship between the residual and initial velocity is depicted as

$$
v_{\text {residual }}=v_{\mathrm{bl}}\left[\left(\frac{v_{\text {initial }}}{v_{\mathrm{bl}}}\right)^{2}-1\right]^{1 / 2} .
$$

Assuming the ballistic velocity is $180 \mathrm{~m} / \mathrm{s}$, the residual velocities for the present penetration simulations can be calculated based on the initial velocities. The calculated residual velocities are compared with the numerical results and the comparison is displayed in Figure 4. From that figure, it can be found that the residual velocities computed from the ISV damage model are closer to the analytical results than the ones yielded from the JC model.

\section{Discussions}

As demonstrated in above sections, both the JC model and the ISV damage model can correctly predict penetration 


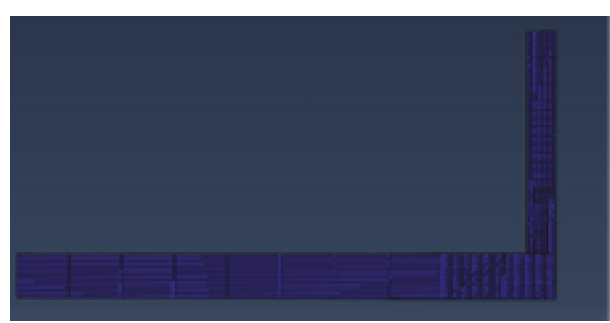

(a)

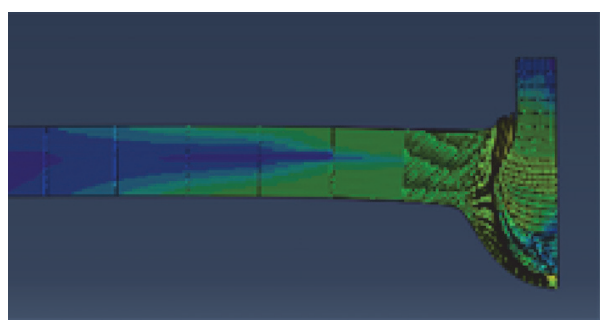

(c)

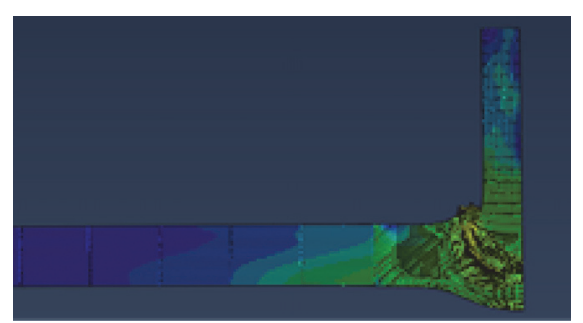

(b)

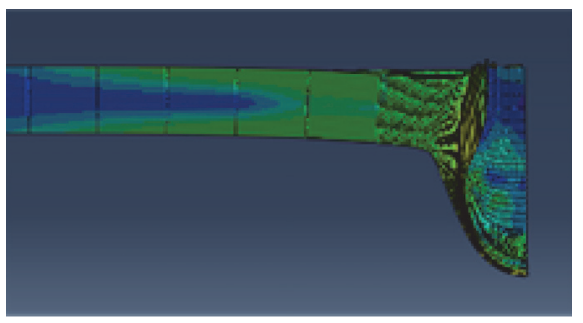

(d)

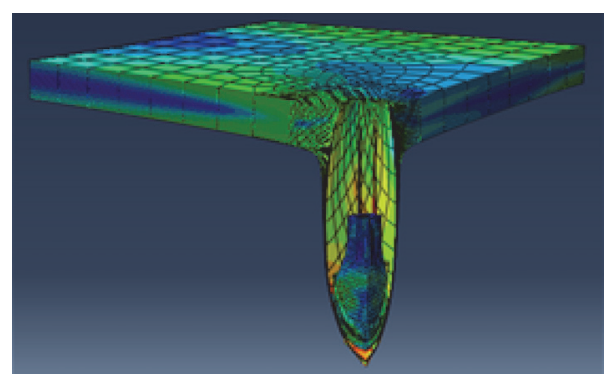

(e)

Figure 5: Penetration simulation yielded from the ISV damage model, $v_{\text {initial }}=200 \mathrm{~m} / \mathrm{s}$ at $t=0$ (a), $50 \mu \mathrm{s}$ (b), $100 \mu \mathrm{s}$ (c), $150 \mu \mathrm{s}$ (d), and $265 \mu \mathrm{s}$ (e).

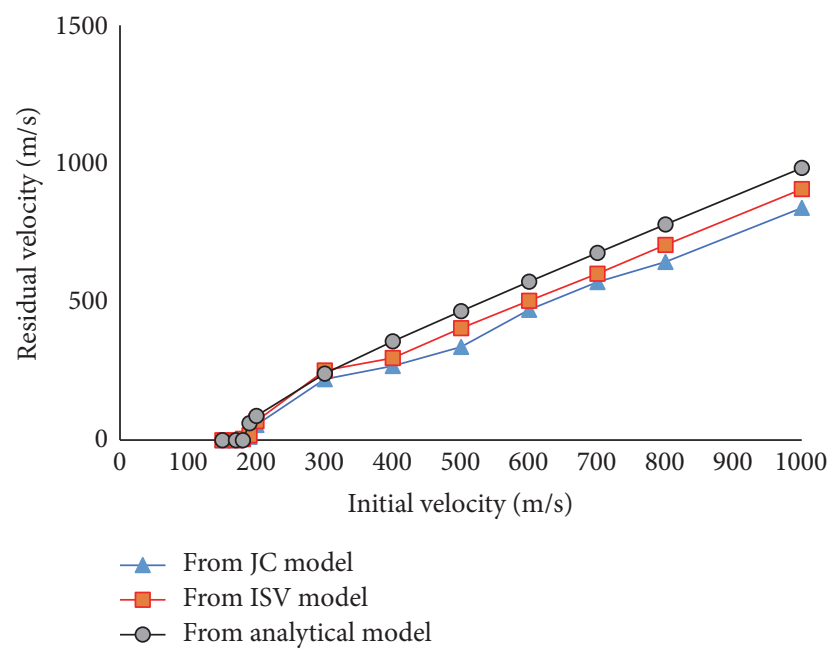

FIGURE 6: Initial velocity versus residual velocity curves from JC, ISV, and analytical models.

mechanics of commonly used metallic materials. The simulation results of the $\mathrm{Al}-\mathrm{Al}$ penetration calculated from both models are very close to each other and the comparison of

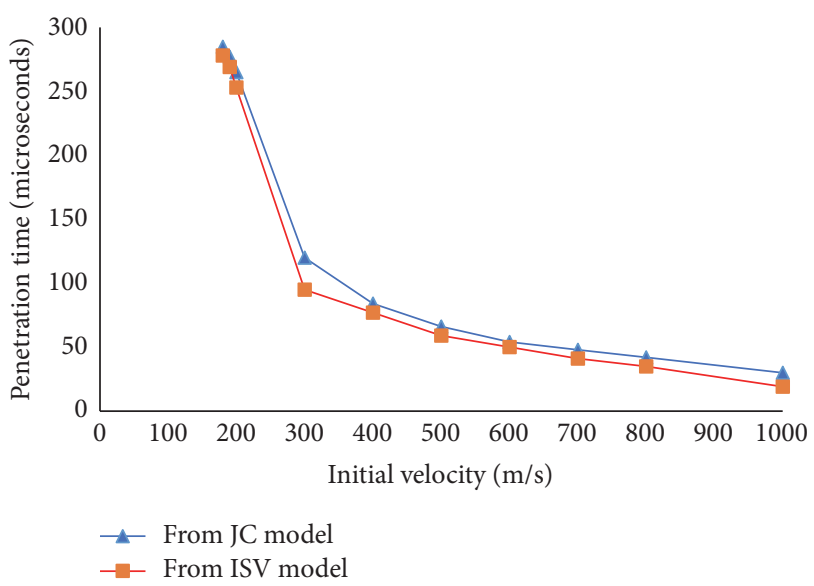

FIGURE 7: Initial velocity versus penetration time curves from JC and ISV models.

those results with the analytical results calculated from a validated plate perforation model is also excellent. Assuming an independent effect of the strain, strain rate, and the temperature, the correlation of the JC model to experimental data is not satisfactory for many materials, especially newly 


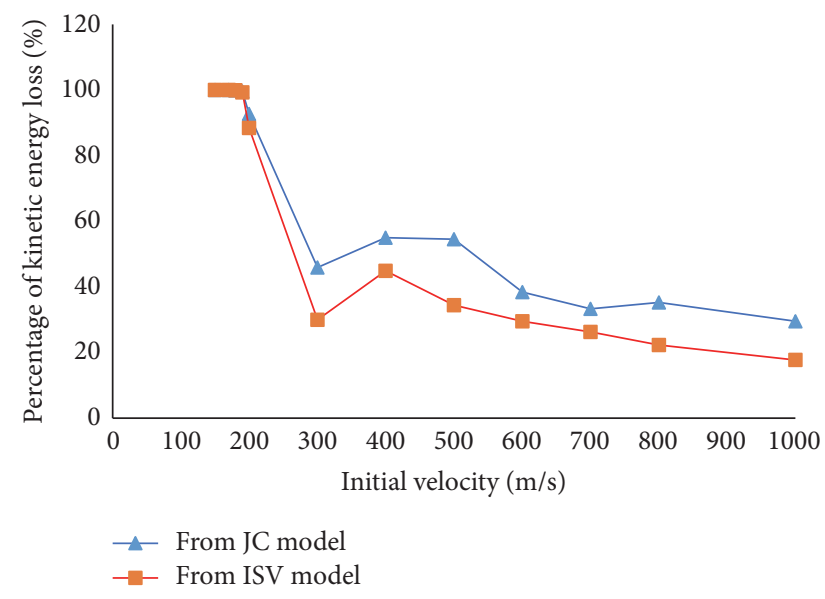

FIGURE 8: Initial velocity versus $v_{\text {initial }}{ }^{2}-v_{\text {residual }}{ }^{2}$ curves from JC and ISV models.

developed materials. Compared to the JC model, the ISV damage model offers some unique merits for simulation of material behavior during high-rate damage process such as high-speed impact and penetration. At first, as shown in (4), (7), and (9)-(11), the ISV model is established based on a set of ISV rate equations, which aim at capturing the history of the changing ISVs involved in a high-rate process and try to use that history to predict a future state. This feature makes the ISV model more applicable for simulation of the penetration process, a process that is characterized by rapidly changed state variables (stress, strain, displacement, etc.), as verified in Figure 4. Secondly, the ISV damage model correlates the global failure and damage behavior of a material to its microstructural features, such as the nucleation, growth, and coalescence of microvoids and microcracks. Therefore, compared to the JC model, whose accuracy largely relies upon a massive amount of data collected from previous penetration tests, the present ISV damage model essentially explains the damage mechanisms of a material in terms of principles and theories in solid mechanics, applied mathematics, and physics. At last, the JC model describes constitutive relations of materials from their stress-strain behavior with strain-rate dependence only and does not consider the effect of different stress states. Contrarily, the ISV damage model presents the constitutive relations by considering both strain rate and stress state. In calibration of the material constants, a series of compression, tension, and torsion tests were conducted at different strain rate and temperature levels in order to maximally reproduce materials' response under complicated stress and strain states, which are often encountered in highspeed impact and penetration. Therefore, compared to the JC model, the ISV damage model can be extensively used for studying penetration mechanics of materials whose penetration performance has not been thoroughly tested through real experiments (e.g., copper, composites, etc.) to promote futuristic design of materials with improved impact and penetration resistance and for simulating the penetration process which cannot be physically tested (e.g., penetration at micro or even atomistic scale) to investigate the length-scale effects on penetration properties of materials and even particles.
The penetration properties of $\mathrm{Al}$ are also revealed on inspecting the simulation results. As reflected in Figures 6-8, as the initial velocity increases, the residual velocity increases as well, the penetration time decreases, and the percentage of kinetic energy lost during the penetration process also decreases. From Figures 2 and 3, it can also be observed that, due to its good ductility, large deformation occurred on the $\mathrm{Al}$ plate before being completely penetrated. That large deformation is due to the flat head of the Al rod, which will be significantly reduced when a rod with a conical head is applied.

\section{Conclusion}

This paper for the first time applies the microstructure-based ISV damage model for simulation of high-speed penetration process. Penetration properties of $\mathrm{Al}$ are investigated using this model through FEA analysis. The simulation results are in good agreement with the results yielded from the JC model and from a validated empirical model. Through comparing the simulation results with the experimental and analytical data, the present study demonstrates the capability of the ISV damage model in capturing and predicting the high-speed impact and penetration process of engineering materials. Compared with traditional JC or other empirical models, the present research introduces a different and more accurate approach to determine mechanical behavior of metals under changing loading situations with loading history effect in the impact and penetration process. The use of many internal state variables enables researchers to (1) track variations of strain, strain rate, temperature, and their history effects on damage constitutive relationship of materials and (2) capture evolutions of microstructure properties such as void and grain size and bridge the development of the microstructure properties to the plastic and damage behavior of materials. Owing to its unique merits, the ISV damage model possesses a bright future in either futuristic design of new materials with improved impact and penetration resistance or the investigation of penetration mechanics of material systems and particles at different length scales.

\section{Disclosure}

This paper is an extension of the conference paper "Computational Investigation of High Velocity Penetration of Copper Subjected to Impact from Nickel Projectiles," which is included in the Proceedings of ASME 2015 International Mechanical Engineering Congress and Exposition with Paper no. IMECE2015-50241.

\section{Competing Interests}

The authors declare that they have no competing interests.

\section{References}

[1] G. R. Johnson and W. H. Cook, "A constitutive model and data for metals subjected to large strains, high strain rates and high temperatures," in Proceedings of the 7th International Symposium on Ballistics, vol. 21, pp. 541-547, 1983. 
[2] G. R. Johnson and W. H. Cook, "Fracture characteristics of three metals subjected to various strains, strain rates, temperatures and pressures," Engineering Fracture Mechanics, vol. 21, no. 1, pp. 31-48, 1985.

[3] S. Dey, T. Børvik, X. Teng, T. Wierzbicki, and O. S. Hopperstad, "On the ballistic resistance of double-layered steel plates: an experimental and numerical investigation," International Journal of Solids and Structures, vol. 44, no. 20, pp. 6701-6723, 2007.

[4] N. Kiliç and B. Ekici, "Ballistic resistance of high hardness armor steels against $7.62 \mathrm{~mm}$ armor piercing ammunition," Materials \& Design, vol. 44, pp. 35-48, 2013.

[5] A. Manes, F. Serpellini, M. Pagani, M. Saponara, and M. Giglio, "Perforation and penetration of aluminium target plates by armour piercing bullets," International Journal of Impact Engineering, vol. 69, pp. 39-54, 2014.

[6] A. Manes, D. Lumassi, L. Giudici, and M. Giglio, "An experimental-numerical investigation on aluminium tubes subjected to ballistic impact with soft core 7.62 ball projectiles," Thin-Walled Structures, vol. 73, pp. 68-80, 2013.

[7] T. Zhang, W. Chen, Y. Guan, D. Gao, and S. Li, "Study on ballistic penetration resistance of titanium alloy TC4, Part II: numerical analysis," Chinese Journal of Aeronautics, vol. 26, no. 3, pp. 606613, 2013.

[8] Y.-Q. Dou, Y.-C. Liu, and Y. Hammi, "Computational investigation of high velocity penetration of copper subjected to impact from nickel projectiles," in Proceedings of ASME 2015 International Mechanical Engineering Congress \& Exposition (IMECE '15-50241), Houston, Tex, USA, November 2015.

[9] E. A. Flores-Johnson, M. Saleh, and L. Edwards, "Ballistic performance of multi-layered metallic plates impacted by a 7.62-mm APM2 projectile," International Journal of Impact Engineering, vol. 38, no. 12, pp. 1022-1032, 2011.

[10] D. J. Bammann, M. L. Chiesa, and G. C. Johnson, "Modeling large deformation and failure in manufacturing processes," in Theoretical and Applied Mechanics, T. Tatsumi, E. Wannabe, and T. Kambe, Eds., pp. 359-376, Elsevier, Amsterdam, The Netherlands, 1996.

[11] Y. Liu, Y. Dou, W. Justin, S. J. Horstemeyer, R. V. K. G. Thirumalai, and W. Williams, "Experimental study of high velocity penetration of an aluminum target plate by a spherical projectile," in Proceedings of the ASME International Mechanical Engineering Congress and Exposition (IMECE '15), ASME, Houston, Tex, USA, November 2015.

[12] Z. Rosenberg and M. J. Forrestal, "Perforation of aluminum plates with conical-nose rods-additional data and discussion," Journal of Applied Mechanics, vol. 55, no. 1, pp. 236-238, 1988.

[13] A. J. Piekutowski, M. J. Forrestal, K. L. Poormon, and T. L. Warren, "Perforation of aluminum plates with ogive-nose steel rods at normal and oblique impacts," International Journal of Impact Engineering, vol. 18, no. 7-8, pp. 877-887, 1996.

[14] Y. Bao and T. Wierzbicki, "On fracture locus in the equivalent strain and stress triaxiality space," International Journal of Mechanical Sciences, vol. 46, no. 1, pp. 81-98, 2004.

[15] T. Demir, M. Übeyli, and R. O. Yildirim, "Investigation on the ballistic impact behavior of various alloys against $7.62 \mathrm{~mm}$ armor piercing projectile," Materials \& Design, vol. 29, no. 10, pp. 2009-2016, 2008.

[16] K. N. Solanki, E. Acar, M. Rais-Rohani, M. F. Horstemeyer, and W. G. Steele, "Product design optimization with microstructure-property modelling and associated uncertainties," International Journal of Design Engineering, vol. 2, no. 1, pp. 47-79, 2009.
[17] R. Carino, M. Horstemeyer, and C. Burton, "Re-engineering DMGFIT-fitting material constants to internal state variable models," MSU.CAVS.CMD.2007-R0040, December 2007.

[18] H. M. Ledbetter and R. P. Reed, "Elastic properties of metals and alloys, I. Iron, nickel, and iron-nickel alloys," Journal of Physical and Chemical Reference Data, vol. 2, no. 3, pp. 531-618, 1973.

[19] Y.-Q. Dou, Y.-C. Liu, W. Whittington, and J. Miller, "Experimental calibration of ISV damage model constants for pure copper for high-speed impact simulation," in Proceedings of the International Mechanical Engineering Congress \& Exposition (ASME '16), IMECE2016-65690, Phoenix, AZ, USA, November 2016.

[20] V. K. Champagne, D. J. Helfritch, M. D. Trexler, and B. M. Gabriel, "The effect of cold spray impact velocity on deposit hardness," Modelling and Simulation in Materials Science and Engineering, vol. 18, no. 6, Article ID 065011, 8 pages, 2010.

[21] Y.-Q. Dou, Y.-C. Liu, and Y. Hammi, "Comparison of JohnsonCook model and an ISV plasticity damage model in penetration simulation," in Proceedings of the ASME International Mechanical Engineering Congress \& Exposition, IMECE201668195, Phoenix, Ariz, USA, November 2016. 


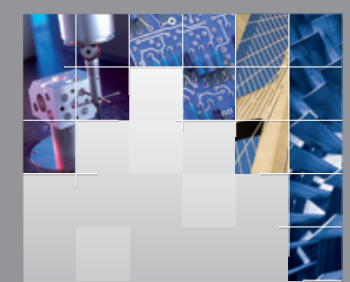

\section{Enfincering}
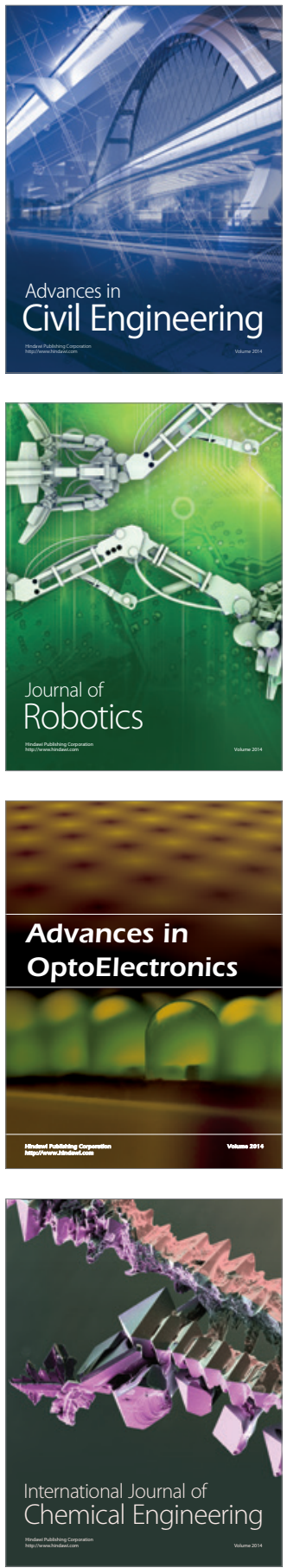

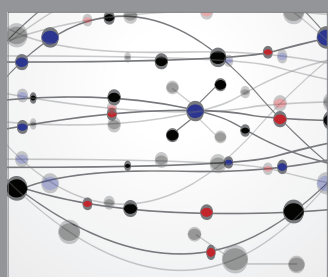

The Scientific World Journal

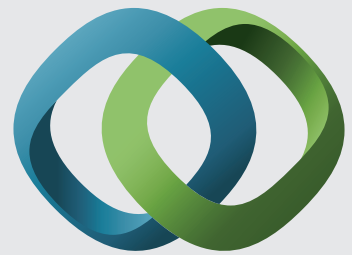

\section{Hindawi}

Submit your manuscripts at

https://www.hindawi.com
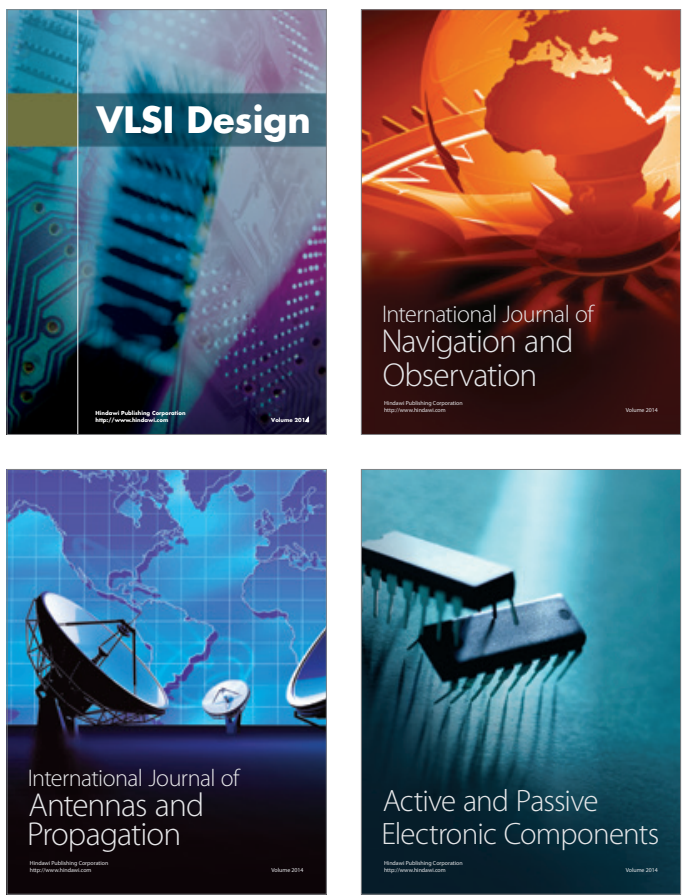
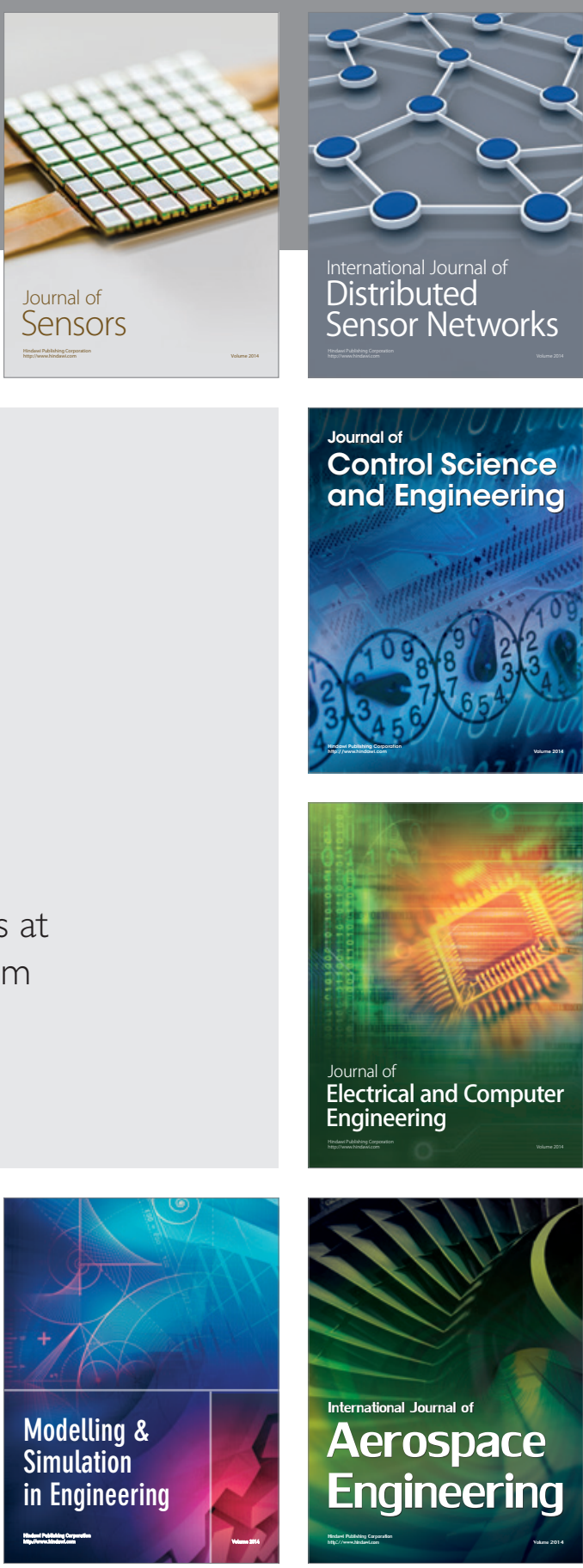

International Journal of

Distributed

Sensor Networks

$-$

Joumal of

Control Science

and Engineering
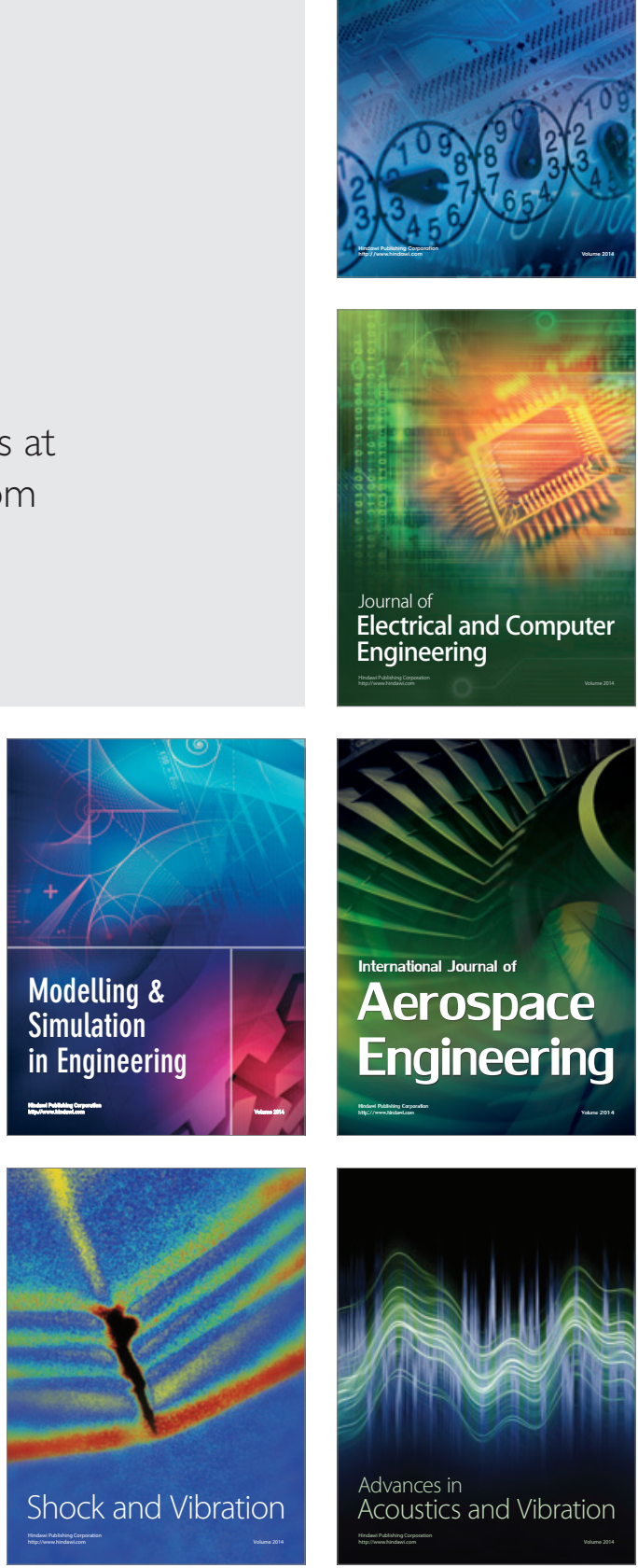